\title{
Magneto-Optic Spatial Light Modulators with Magnetophotonic Crystals Driven by PZT Films
}

\author{
H. Takagi ${ }^{*, * *}$, J. Kim*, K. H. Chung*, S. Mito*, H. Umezawa***, and M. Inoue* \\ * Toyohashi University of Technology, 1-1 Hibari-Ga-Oka, Tempaku, Toyohashi, Aichi 441-8580, Japan \\ ${ }^{* *}$ Toyota National College of Technology, 2-1 Eisei, Toyota, Aichi 471-8525, Japan \\ ${ }^{* * *}$ FDK Corporation, 2281 Washidu, Kosai, Shizuoka, 431-0495, Japan
}

Spatial light modulator (SLM) is a real-time programmable device for modifying amplitude, phase or polarization of optical wave front by electrically controlled signals. Various types of SLMs with pixel arrays, such as a liquid crystal SLM, have been developed. Recently, SLMs have received much attention as a light modulator in holographic data storage which requires high speed SLMs for ensuring the high data transfer rate. In this field, the application of a magneto-optic SLM (MOSLM) is highly expected because of extremely fast pixel switching speed. The conventional MOSLM used a single crystal garnet with perpendicular magnetic anisotropy to switch as a binary depending on up and down magnetization. However, for an analog modulation in our concept, the direction of magnetization is gradually changed to in-plane direction, because the modulation is depending on the magnetization direction of the garnet film. A voltage driven MOSLM for analog modulation is developed using magnetostriction effect. Moreover, the MOSLM with a structure of a magnetophotonic crystal (MPC) is fabricated for the large enhancement of magneto-optical response. This report is about a current development of MOSLMs with magnetophonic crystal driven by a voltage.

Key words: Magneto-optic spatial light modulator, Garnet, Magnetophotonic crystal, Magneto-optical response

\section{Introduction}

Spatial light modulator (SLM) is a real-time micro-device for modulating the amplitude (or intensity), phase, or polarization of optical waves as a function of the spatial position across the wavefront. SLMs with liquid crystal or MEMS technology, such as DMD or GLV, are now commercially available, and are widely used as a key component in image projection systems. Recent needs of more sophisticated SLMs enabling ultra-high speed modulation of light have resulted from the renewed interests in holographic data storage or 3-dimensional display, which fields require high rate of data transfer. For such applications, SLMs based on the magneto-optical effect (Magneto-optic SLMs, MOSLMs) are to be a good candidate ${ }^{1)-4)}$. The reason is that MOSLMs provide magnetic visual images based on the Faraday effect, and the pixel switching takes place by the magnetization reversal being inherently very fast in the order of several nanoseconds. Non-volatility, robustness and radioactive resistance are also the main features of MOSLMs. In this paper, our historic researches of the MOSLM are reviewed and recent developments of the MOSLM are described in term of a structure of magnetophotonic crystals and driving of piezoelectric effect.

\section{MOSLMs with LPE garnet films:} $i$-MOSLM and $v$-MOSLM

Figure 1 shows a typical photograph of the MOSLM driven by currents ( $i$-MOSLM) for commercialization. The device contains $128 \times 128$ magnetic pixels with a liquid phase epitaxy (LPE)-grown garnet film, where the pixels with $14 \times 14 \mu \mathrm{m}$ square shape were simultaneously formed during the LPE garnet formation. Switching speed of each pixel of this MOSLM is approximately $15 \mathrm{~ns}$, considerably high speed as a solid state SLM.

Typical performance of the device is summarized as the following: Faraday rotation is \pm 8.5 degrees at $532 \mathrm{~nm}$, extinction ratio less than $-30 \mathrm{~dB}$, optical reflectivity of $21.7 \%$, and fill factor of $76.6 \%$.

In $i$-MOSLM, pixels are driven by currents which average intensity is several ten $\mathrm{mA}$. For operating a large number of pixels such as $1024 \times 1024$, however, the total current intensity required for the device operation will result in a serious heat problem of the devices. To avoid heating up, voltage-driven MOSLM ( $v$-MOSLM) was designed and tested its fundamental performance. $v$-MOSLM has a layered structure composed of a LPE-magnetic garnet film and a $\mathrm{Pb}_{1}(\mathrm{Zr}, \mathrm{Ti})_{1} \mathrm{O}_{3} \quad$ (PZT) piezoelectric film, so as to achieve the magnetization switching not by magnetic fields from current but by piezoelectrically induced stresses from the PZT film to the garnet film. Switching mechanism for magnetization in the Bi:YIG pixel of the $v$-MOSLM is shown in Fig. 2. The applied stresses tend to be effective magnetic field for magnetization switching via magnetostriction of garnet $^{5)-6)}$. Then, $v$-MOSLM employs apparent multiferroic operation, where the control of magnetization is achieved by a voltage applied to the PZT film. This is attractive for reducing the intensity of large current for a number of pixels. Based upon the principle, we fabricated a prototype $v$-MOSLM with the same garnet film as used in $i$-MOSLM. Fig. 3 shows the results of the threshold external magnetic field for the magnetic switching in a pixel as a function

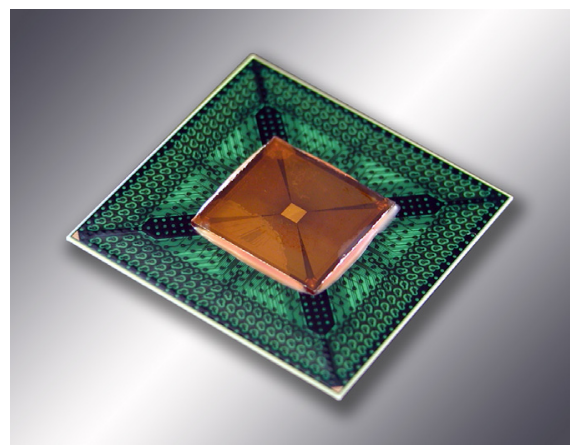

Fig. 1 Current-driven MOSLM with $128 \times 128$ pixels (manufactured by FDK). 


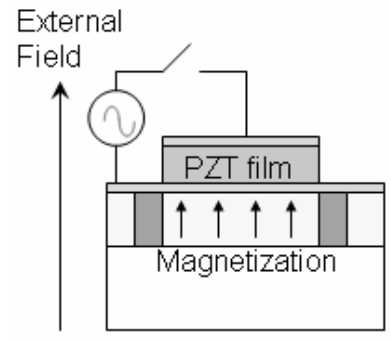

(a)

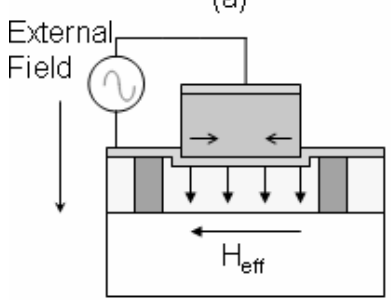

(c)

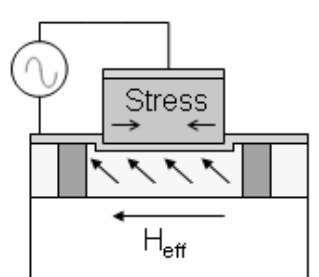

(b)

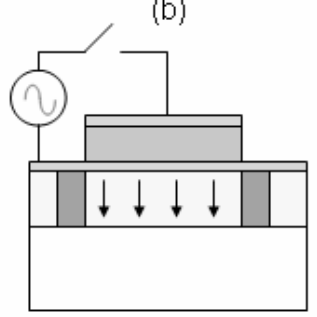

(d)
Fig. 2 Mechanism of switching magnetization in the magneto-optical Bi:YIG layer. (a) The first step of magnetization saturated by the external bias field, (b) the second step of applying a voltage to the piezoelectric PZT film, (c) the third step of applying the small external bias field for complete switching and (d) the final step of switching magnetization without a voltage.

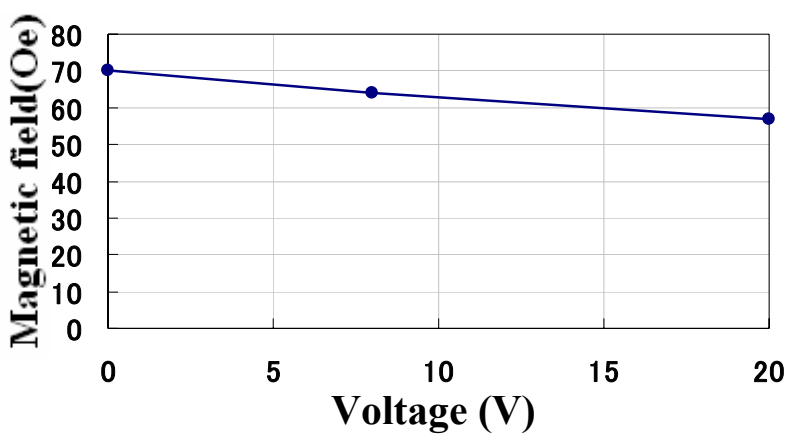

Fig. 3 Change of threshold external magnetic field for switching the pixels of MOSLM as a function of an applied voltage to the PZT layer.

of an applied voltage to the PZT film for stress. As the voltage was increased, the magnetic field became smaller because of inverse magnetostriction of the garnet film. By applying $20 \mathrm{~V}$ for a designated pixel, the hybrid structure was found to be effective for reducing about $18 \%$ of the intensity of external bias field, where the drive current intensity was considerably small ranging from 200 to $500 \mathrm{nA}$.

To enhance the device performance, use of magnetic garnet films with large magnetostriction without lowering of the good magneto-optical response is indispensable.

\section{Analog modulation MOSLMs}

These above MOSLMs were made of a LPE-garnet film with strong magnetic anisotropy along the direction of out-of-plane; therefore, binary modulation depending on up or down direction of magnetization.

When the direction of magnetization is changed from easy-axis to hard-axis, the intensity of light can be gradually changed. Based on this concept, we tried to fabricate a new MOSLM for an analog modulation with high speed $^{7)}$. The driving principle was demonstrated by external magnetic field.

We used a polycrystalline garnet film with small magnetocrystalline anisotropy fabricated by rf magnetron sputtering method.

Figure 4 shows change of brightness in pixels as a function of external magnetic field, which was obtained by a polarization microscope with a charge coupled device (CCD) camera. The brightness was increased in proportion to the external magnetic field, which inclination was about 30 a.u./kOe between magnetic field of -1.2 and $1.2 \mathrm{kOe}$.

In this case, necessary magnetic field is very large for the analog modulation. The large driving current for magnetic field must be keep to apply to be constant grayscale level. The developed analog modulation $v$-MOSLMs, which will be discussed in the next section, showed the low power consumption for driving, because of the steady state of PZT behavior. The stress by piezoelectric effect plays a role as the effective magnetic field, which is depending on the direction magnetization of the garnet film.

\section{MOSLMs with magnetophotonic crystals}

A thin film type MOSLM with one-dimensional magnetophotonic crystals (1D-MPCs) has developed. As shown in Fig.5, 1D-MPC has a micro-cavity structure, where a thin magnetic garnet film is sandwiched between two Bragg mirrors or metal mirror. Due to the strong localization of light, largely enhanced Faraday rotation (Fig.6) exhibits at the wavelength of the resonance reflectivity of light ${ }^{8)}$. By employing the $v$-MOSLM technology, direction of the magnetization is controlled between perpendicular and in-plane. This means that the modulation of circularly polarized light is possible, because the phase of light in such a magnetic pixel depends on the relative directions of light propagation and magnetization ${ }^{10)}$.

As shown in Fig. 7, the MPC has a reflection-type micro-cavity structure, where the $\mathrm{Dy}_{1.0} \mathrm{Bi}_{1.5} \mathrm{Y}_{1.0} \mathrm{Fe}_{5} \mathrm{O}_{12}$ (DyBi:YIG) film deposited using rf ion beam sputtering was sandwiched between $\mathrm{Ag}$ bottom electrode and $\left(\mathrm{SiO}_{2} / \mathrm{Ta}_{2} \mathrm{O}_{5}\right)^{2}$ Bragg mirror layer using a glass substrate. The thickness of the layers was designed to localize the light. The top layer of the MPC structure was fabricated by metal mirror, Au on the garnet film, because of close position between PZT film and the garnet film.

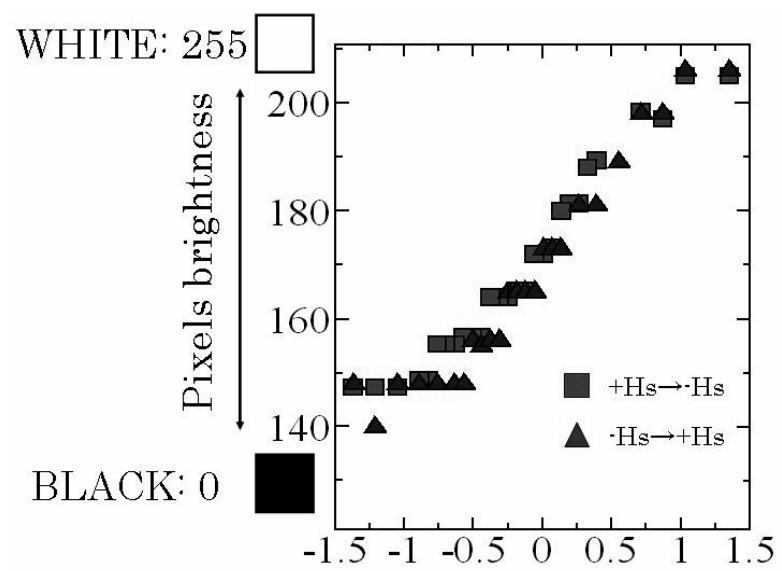

Magnetic external field ( $\mathrm{kOe})$

Fig. 4 Change of brightness of pixels in the analog modulation MOSLM measured by a polarization microscope with a CCD camera. In the camera image, maximum was 255 A.U. and minimum was 0 A.U. in brightness. 
PZT film with a thickness of $2 \mu \mathrm{m}$ was deposited by means of aerosol deposition method ${ }^{9)}$. The PZT film was annealed at 500 ${ }^{\circ} \mathrm{C}$ for 30 minutes for re-crystallization. The upper Au electrode (300 nm thick) was patterned by the lift-off process.

In the structure of MPC, figure 8 shows the variation of Kerr rotation at the localized wavelength by applying voltage to PZT film. Kerr rotation angle was changed in proportion to the applied voltage to PZT film, such as, 0.2 degree deviation with applying voltage of $20 \mathrm{~V}$. It meant that the magnetization direction of the garnet film was controlled by stress from the PZT film. In other words, stress played a role of magnetic field of in-plane direction to change the direction of out-of-plane magnetization. These values showed a good agreement with the calculated effective magnetic field. A stoichiometrical optimization of the magnetic garnet in terms of magneto-optical and magnetic properties and magnetostriction and a modification of MPC structure are the key factors for higher performance.

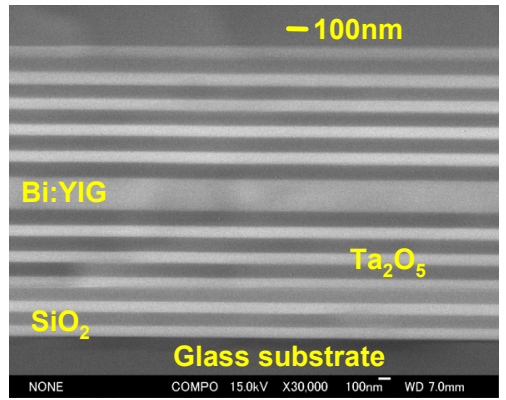

Fig. 5 Cross-sectional SEM image of microcavity type 1D-MPC with a thin magnetic garnet film.

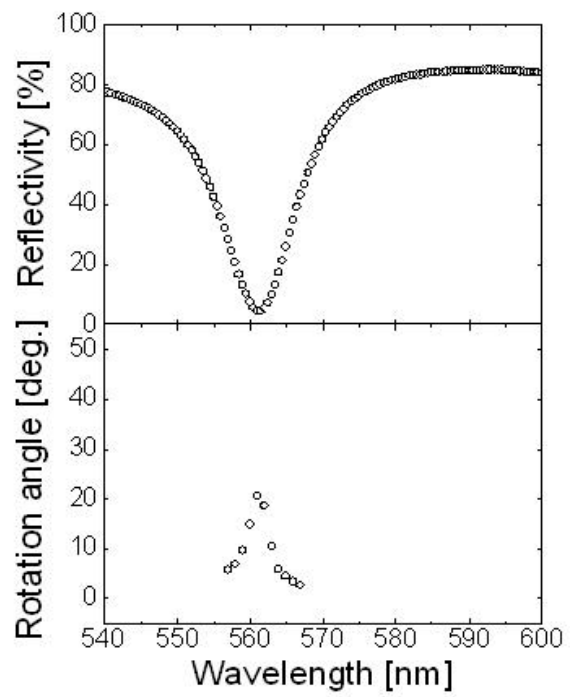

Fig.6 Optical and magneto-optical properties of micro-cavity 1D- MPC.

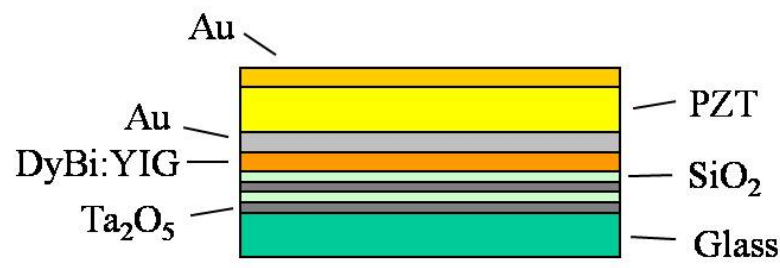

Fig. 7 Structure of newly designed MPC-MOSLM

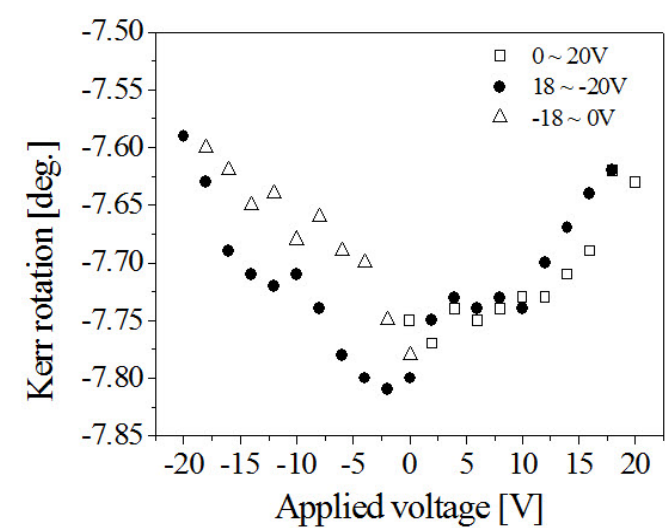

Fig. 8 Variation of Kerr rotation of MPC-MOSLM with applied voltage.

\section{Conclusion}

SLMs have received much attention as a light modulator in holographic data storage which requires high speed SLMs for ensuring the high data transfer rate. In this field, the application of a magneto-optic SLM (MOSLM) is highly expected because of extremely fast pixel switching speed.

The commercial current driven MOSLM is faced to be released commercially. The voltage driven MOSLM has been developed and succeeded to decrease power consumption. Currently, the voltage driven analog modulation MOSLM with MPC structure is investigated. In addition, the application of MOSLMs is opened in optical field for the requirement of high modulation speed, such as a collinear phase-lock holography, an optical correlator and a three-dimensional display.

Acknowledgements The work was supported in part by the Super Optical Information Memory Project, MEXT, and also by the Grant-in-Aid for Scientific Researches S (No.17106004), JSPS.

\section{References}

1) J. K. Cho, S. Santhanam, T. Le, K. Mountfield, D. N. Lambeth, and D. Stancil, J. Appl. Phys., 761910 (1994).

2) W. E. Ross, J. P. Karins, T. Maki, J. Lucas, L. G. Kelly, J. Cho, D. N. Lambeth, T. Le, K. Mountfield, S. Santhanam, D. Stancil, M. Randles, J. Whitlock, and D. Garrity, Proc. SPIE, 1959, 222 (1993).

3) J. H. Park, J. K. Cho, and M. Inoue, Jpn. J. Appl. Phys., 41, 3B, 1813 (2002).

4) J. H. Park, H. Takagi, J. Park, and J. K. Cho, K. Nishimura, H. Uchida and M. Inoue, J. Appl. Phys., 93, 8525 (2003).

5) H. Takagi, K. Takahashi, S. Mito, F. Kawanishi, K. H. Chung, J. Heo, J. Kim, P. B. Lim, M. Inoue, J. Magn. Soc. Jpn., 30(6-2), 581 (2006).

6) K. Schröder, J. Appl. Phys., 53, 2759 (1982).

7)A. Tsuzuki, H. Takagi, P. B. Lim, H. Uchida, K. H. Shin, M. Inoue, J. Magn. Soc. Jpn., 30(6-2), 571 (2006).

8)K. Takahashi, H. Takagi, K. H. Shin, H. Uchida, P. B. Lim, M. Inoue, J. Appl. Phys., 101, 09C523 (2007).

9)M. Levedev, J. Akedo, Y. Akiyama, Jpn. J. Appl. Phys., Part 1, 39(9B), $5600(2000)$.

10)K. H. Chung, J. Heo, K. Takahashi, S. Mito, H. Takagi, J. Kim, P. B. Lim, M. Inoue, J. Magn. Soc. Jpn., 32(2-2), 114 (2008).

Received Jul. 13, 2009; Received Sep. 4, 2009; Accepted Sep. 8, 2009 